\title{
Correction to: Entropy in a Closed Manifold and Partial Regularity of Mean Curvature Flow Limit of Surfaces
}

\author{
Ao Sun $^{1}$ iD \\ Published online: 22 May 2021 \\ (c) Mathematica Josephina, Inc. 2021
}

\begin{abstract}
We correct some mistakes in "Entropy in A Closed Manifold and Partial Regularity of Mean Curvature Flow Limit of Surfaces".

\section{Correction to: The Journal of Geometric Analysis https://doi.org/10.1007/s12220-020-00494-z}

There is a computational mistake in the original paper, (4.5). As a consequence, we can not have finiteness of entropy in a closed manifold, i.e. Theorem 1.6 may not be true in a compact manifold. Nevertheless, the final conclusion of the original paper does still hold, which I would like to explain in the following. I want to thank Pedro Gasper for pointing out the mistakes.

I will use the notations in the original paper. In the proof of Theorem 1.2 (see the end of Sect. 6), where we need to use the fact that that the area growth $\kappa$ of $M_{t}$ is uniformly bounded is the usage of Theorem 5.5. However, the consequence of Theorem 5.5 holds even we do not have initial entropy bound. The reason is that, the only place where we use area growth bound is in Corollary 5.3, and the only place where we use area growth bound in Corollary 5.3 is in Theorem 5.1. In Theorem 5.1, the area growth bound appears in the statement of the theorem: we need the area upper bound $\operatorname{Area}\left(M \cap B_{R}\right) \leq D \pi R^{2}$.

In the original paper, we need to use entropy to control this $D$. However, for the purpose of the proof of Theorem 1.2, we do not need to use entropy to control $D$. Instead, we only need to bound $D$ by $\operatorname{Area}(M) /\left(\pi R^{2}\right)$. Namely, once the surface $M$ has finite area, we can have a control of $D$. Though this bound is very crude, it is suffices to prove Theorem 1.2. So the original proof of Theorem 1.2 does hold. Similarly,

The original article can be found online at https://doi.org/10.1007/s12220-020-00494-z.

$凶$ Ao Sun

aosun@mit.edu

1 Department of Mathematics, Massachusetts Institute of Technology, Cambridge, MA 02139, USA 
Theorem 1.4 also does hold, if we replace the entropy upper bound assumption by area upper bound asssumption in the statement.

Finally, I want to point out that, although we do not need the entropy upper bound for the proof of Theorem 1.2 and Theorem 1.4, Theorem 1.6 is still true with a minor modification. If the ambient manifold $\mathcal{N}^{n}$ satisfies

- complete, with nonnegative sectional curvature and parallel Ricci curvature,

- $\operatorname{Vol}\left(\mathcal{N} \cap B_{R}\right) \geq C R^{n-m+\epsilon}$, for some $\epsilon>0$,

then the entropy of a closed $m$-dimensional submanifold is still finite, and (1.3) is true. In fact, if $\mathcal{N}$ satisfies the the above two conditions, we may repeat most of proof of Theorem 1.6, but we no longer need to prove for two cases, but only need to proceed the proof of the second case, without assuming $r<r_{i n} / 2$. What we need is directly use Theorem 4.2 and the volume bound assumption of $\mathcal{N}$ listed above.

Publisher's Note Springer Nature remains neutral with regard to jurisdictional claims in published maps and institutional affiliations. 